\title{
Physiological Responses of Nile Tilapia, Oreochromis niloticus, Fed Vitamin C- and Lipid-Supplemented Diets and Submitted to Low-Temperature Stress*
}

\author{
DARIO R. FALCON ${ }^{1}$ \\ AquaNutri, Faculty of Veterinary Medicine and Animal Science, São Paulo State University, \\ Botucatu, CEP 18.618-000, São Paulo, Brazil \\ Margarida M. Barros and Luiz E. Pezzato \\ Faculty of Veterinary Medicine and Animal Science, \\ Breeding and Animal Nutrition Department, São Paulo State University - UNESP, Botucatu, \\ São Paulo CP 560 CEP 18.618-000 Brazil \\ FERNANDA G. SAMPAIO \\ São Carlos Federal University, São Carlos, Brazil \\ HAMILTON HisANO \\ Embrapa, São Paulo, Brazil
}

\begin{abstract}
Water temperature alterations can determine harmful physiological modifications in fish, which should be prepared to cope with this, and nutrition strategies seem to be essential. This study evaluated the effects of different levels of vitamin $\mathrm{C}$ and lipids on physiological responses of Nile tilapia, Oreochromis niloticus, submitted to temperature stress. There were two phases: Phase I - preparing fish to store vitamin $\mathrm{C}$ and lipid at appropriate temperature, and Phase II - evaluating the contributions these reserves make to fish physiology under low-temperature stress. The experiment used a $3 \times 2$ factorial design with three vitamin C levels $(300,600$, and $1200 \mathrm{mg} / \mathrm{kg}$ diet) and two lipid levels $(8.0$ and $12.0 \%)$, plus absence of nutrient test and a diet of $6.0 \%$ lipids and $125.0 \mathrm{mg} / \mathrm{kg}$ vitamin C. In Phase I, 192 fish were kept at $26.0 \pm 1.0 \mathrm{C}$ for $112 \mathrm{~d}$, and in Phase II, 48 fish were kept at $18.0 \pm 0.5 \mathrm{C}$ for $32 \mathrm{~d}$ and at $15.0 \pm 0.5 \mathrm{C}$ for $11 \mathrm{~d}$. Fish fed $\mathrm{C}_{0} \mathrm{~L}_{0}$ diet showed lower erythrocytes values in both phases; higher vitamin $\mathrm{C}$ supplement determined higher red blood cell (RBC) number and higher hematocrit (Htc) (Phase II); Htc was significantly lower in Phase II; after temperature stress, fish fed $\mathrm{C}_{0} \mathrm{~L}_{0}$ diet had higher mean corpuscular volume, lower hemoglobin corpuscular concentration, and significantly lower vitamin C concentration in the liver; and higher supplementation determined a higher concentration in the liver (Phases I and II). Higher plasmatic cortisol concentration was seen in fish fed $\mathrm{C}_{0} \mathrm{~L}_{0}$ diet. In conclusion, our results show that the absence of vitamin $\mathrm{C}$ in diets impairs $\mathrm{RBC}$ formation and does not enable fish to cope with stress; excess vitamin $\mathrm{C}$ is efficient in mitigating stress and

\footnotetext{
${ }^{1}$ Corresponding author.

* Use of trade or manufacturer's name in this article does not imply endorsement.
}

$600 \mathrm{mg} / \mathrm{kg}$ diet is economic and physiologically sufficient to prepare fish for coping with low-temperature stress. Lipid supplementation does not determine alterations in stress biochemical parameters.

In intensive culture systems, fish are continuously exposed to stress, which can cause temporary homeostasis modifications leading to physiological adjustments. These responses aimed at mobilizing energy by adrenergic system stimulation, release catecholamine and increase adrenocorticotropic hormone (ACTH) and plasmatic cortisol (Gamperl et al. 1994). These responses can be extended and cause chronic stress, which increases the imbalance.

Low resistance and high disease susceptibility during a stress period, such as winter, can cause fish death and, consequently, economic problems to producers. According to Sealey et al. (1997), the annual loss from disease problems caused mainly by Edwardsiella ictaluri bacteria is estimated to be millions of dollars in the American catfish industry.

Weight loss has also been observed during winter as a result of body fat reduction (Lovell and Sirikul 1974). According to Lemly (1996), lipid reserves have significant mobilization and can be totally used as an energy source, indicating the need for an appropriate reserve to maintain good body condition. 
The increase in metabolism during adverse situations also determines vitamin redistribution and higher demand, especially vitamin C (Wedemeyer 1969). Although some research showed that higher vitamin $\mathrm{C}$ doses than required for normal growth have been recommended for better resistance in channel catfish, Ictalurus punctatus ( $\mathrm{Li}$ and Lovell 1985), others showed that high doses were not effective ( $\mathrm{Li}$ et al. 1998).

Studies have shown that high ascorbic acid concentration in tissue determines higher tolerance to ambient pollution and better resistance to bacteria infection (Li and Lovell 1985). Jaffa (1989) also recommended that high levels of vitamin C supplementation minimize physiological stress in fish. Kitabchi (1967) stated that high ascorbic acid levels inhibit steroid synthesis, suggesting that an increased vitamin $\mathrm{C}$ reserve might prevent stress response severity.

This study evaluated the effects of different vitamin $\mathrm{C}$ and lipid levels and their interaction on physiological responses of Nile tilapia, Oreochromis niloticus, submitted to low-temperature stress.

\section{Material and Methods}

There were two phases: Phase I (112 d) prepared fish vitamin $\mathrm{C}$ and lipid reserves at an appropriate temperature and Phase II ( $36 \mathrm{~d})$ evaluated the contributions these reserves made to fish physiological responses when submitted to low-temperature stress.

\section{Experimental Diets and Design (Phases I and II)}

The experiment used a $3 \times 2$ factorial design for both phases, with three vitamin $\mathrm{C}(\mathrm{mg} / \mathrm{kg}$ diet) and two lipid (\%) levels: $300 \mathrm{mg}, 8 \%\left(\mathrm{C}_{300} \mathrm{~L}_{8}\right)$; $600 \mathrm{mg}, 8 \%\left(\mathrm{C}_{600} \mathrm{~L}_{8}\right) ; 1200 \mathrm{mg}, 8 \%\left(\mathrm{C}_{1200} \mathrm{~L}_{8}\right)$; $300 \mathrm{mg}, \quad 12 \% \quad\left(\mathrm{C}_{300} \mathrm{~L}_{12}\right) ; \quad 600 \mathrm{mg}, \quad 12 \%$ $\left(\mathrm{C}_{600} \mathrm{~L}_{12}\right)$; and $1200 \mathrm{mg}, 12 \%\left(\mathrm{C}_{1200} \mathrm{~L}_{12}\right)$. Two other treatments were added: a nonsupplemented $\left(\mathrm{C}_{0} \mathrm{~L}_{0}\right)$ and a $125 \mathrm{mg}, 6 \%\left(\mathrm{C}_{125} \mathrm{~L}_{6}\right)$. Polyphosphated vitamin $\mathrm{C}$ with $35 \%$ ascorbic acid activity (Stay-C ${ }^{\circledR}$ Roche Socil, Brazil) was used with commercial soybean oil as the lipid source.
Eight practical diets were formulated containing approximately $32.0 \%$ digestible protein (Table 1). All ingredients were ground to pass through a 1-mm mesh screen and processed into 3-mm-diameter pellets. Vitamin and mineral supplements did not contain vitamin C. Diets were dried and frozen $(-20.0 \mathrm{C})$ to avoid vitamin $\mathrm{C}$ loss as a result of oxidation. Crude protein, crude energy, and ether extract were chemically analyzed according to AOAC (2000) protocol. Dietary vitamin C levels were assessed by high-performance liquid chromatography (HPLC). Feed samples (1.0-2.0 g) were ground to a fine powder, extracted by ultrasound bath for $15 \mathrm{~min}$, cooled, and then filtered in millex $(0.45 \mu \mathrm{m})$. Chromatography conditions were as follows: mobile phase flux $2.0 \mathrm{~mL} / \mathrm{min}$, ODS column $(\mathrm{C} 18$ $150 \times 4.6 \mathrm{~mm}), 25 \mathrm{C}$, and $280-\mathrm{nm}$ UV detector. Detection level was about $10 \mathrm{ppm}$, and L-ascorbyl-2-polyphosphate recovery efficiency was $94 \%$.

\section{Phase I - Fish and Feeding}

In Phase I, 192 Nile tilapia fingerlings from a single spawn and sex reverted (methyltestosterone treated), with an average weight of $5.57 \pm 0.50 \mathrm{~g}$, were randomly distributed into 32 net cages $(200 \mathrm{~L}$ each), four cages per treatment and six fish per cage, placed in eight $1000-\mathrm{L}$ aquaria in a closed recirculation system. Aquaria were supplied with $6 \mathrm{~L} / \mathrm{min}$ dechlorinated tap water passing through a biofilter to remove impurities and reduce ammonia concentration. The system was supplied with a heater and kept at $26.0 \pm 1.0 \mathrm{C}$. Water temperature and dissolved oxygen were measured once a week in four randomly selected tanks using a DM4 oxygen meter (DIGIMED Adamo, Brazil); accumulated wastes were removed by siphoning.

Each of the eight experimental diets was randomly fed to fish in four net cages four times a day $(0830,1130,1430$, and $1730 \mathrm{~h})$ for $112 \mathrm{~d}$. At each feeding, diet was offered two or three times until apparent satiation was reached. A 12-h photoperiod was maintained with artificial fluorescent illumination. 
TABLE 1. Proximal and chemical composition of experimental diets.

\begin{tabular}{|c|c|c|c|c|c|c|c|c|}
\hline \multirow[b]{2}{*}{ Ingredient } & \multicolumn{8}{|c|}{ Diets $^{a}$} \\
\hline & $\mathrm{C}_{0} \mathrm{~L}_{0}$ & $\mathrm{C}_{125} \mathrm{~L}_{6}$ & $\mathrm{C}_{300} \mathrm{~L}_{8}$ & $\mathrm{C}_{600} \mathrm{~L}_{8}$ & $\mathrm{C}_{1200} \mathrm{~L}_{8}$ & $\mathrm{C}_{300} \mathrm{~L}_{12}$ & $\mathrm{C}_{600} \mathrm{~L}_{12}$ & $\mathrm{C}_{1200} \mathrm{~L}_{2}$ \\
\hline Soybean meal & 54.88 & 56.27 & 56.74 & 56.74 & 56.80 & 57.65 & 57.08 & 57.70 \\
\hline Corn gluten meal & 5.00 & 5.00 & 5.00 & 5.00 & 5.00 & 5.00 & 5.00 & 5.00 \\
\hline Fish meal & 8.00 & 8.00 & 8.00 & 8.00 & 8.00 & 8.00 & 8.00 & 8.00 \\
\hline Corn meal & 24.10 & 16.67 & 14.15 & 14.07 & 13.84 & 9.24 & 9.13 & 8.94 \\
\hline Wheat middlings & 5.00 & 5.00 & 5.00 & 5.00 & 5.00 & 5.00 & 5.00 & 5.00 \\
\hline Soybean oil & - & 6.00 & 8.00 & 8.00 & 8.00 & 12.00 & 12.00 & 12.00 \\
\hline Dicalcium phosphate & 2.50 & 2.50 & 2.50 & 2.50 & 2.50 & 2.50 & 2.50 & 2.50 \\
\hline Vitamin-mineral mix ${ }^{b}$ & 0.50 & 0.50 & 0.50 & 0.50 & 0.50 & 0.50 & 0.50 & 0.50 \\
\hline $\mathrm{BHT} \mathrm{c}$ & 0.02 & 0.02 & 0.02 & 0.02 & 0.02 & 0.02 & 0.02 & 0.02 \\
\hline Vitamin $C^{d}$ & - & 0.04 & 0.09 & 0.17 & 0.34 & 0.09 & 0.17 & 0.34 \\
\hline \multicolumn{9}{|l|}{$\begin{array}{l}\text { Chemical composition } \\
\text { (dry matter basis) }\end{array}$} \\
\hline $\mathrm{CP}(\%)^{\mathrm{e}}$ & 36.05 & 36.12 & 35.70 & 34.97 & 35.69 & 36.20 & 36.50 & 36.03 \\
\hline $\mathrm{CE}(\mathrm{kcal} / \mathrm{kg})^{\mathrm{e}}$ & 4640.10 & 4702.04 & 4809.71 & 4858.14 & 4853.41 & 5192.06 & 5184.51 & 5157.60 \\
\hline $\mathrm{EE}(\%) \mathrm{e}$ & 2.79 & 8.88 & 10.89 & 10.81 & 10.68 & 14.59 & 14.44 & 14.68 \\
\hline $\mathrm{CF}(\%)^{\mathrm{f}}$ & 4.28 & 4.19 & 4.16 & 4.16 & 4.16 & 4.12 & 4.12 & 4.12 \\
\hline Vitamin C $(\mathrm{mg} / \mathrm{kg})^{\mathrm{e}}$ & ND & 189.00 & 360.00 & 527.00 & 1247.00 & 331.00 & 493.00 & 1108.00 \\
\hline
\end{tabular}

$\mathrm{BHT}=$ butylated hydroxytoluene; $\mathrm{CP}=$ crude protein; $\mathrm{CE}=$ crude energy; $\mathrm{EE}=$ ether extract; $\mathrm{CF}=$ crude fiber; $\mathrm{ND}=$ not determined.

a Treatments: $\mathrm{C}_{0} \mathrm{~L}_{0}\left(0 \mathrm{mg} / \mathrm{kg}\right.$ vitamin $\mathrm{C}$ and $0 \%$ lipid); $\mathrm{C}_{125} \mathrm{~L}_{6}\left(125 \mathrm{mg} / \mathrm{kg}\right.$ vitamin $\mathrm{C}$ and $6 \%$ lipid); $\mathrm{C}_{300} \mathrm{~L}_{8}(300 \mathrm{mg} / \mathrm{kg}$ vitamin $\mathrm{C}$ and $8 \%$ lipid); $\mathrm{C}_{600} \mathrm{~L}_{8}\left(600 \mathrm{mg} / \mathrm{kg}\right.$ vitamin $\mathrm{C}$ and $8 \%$ lipid); $\mathrm{C}_{1200} \mathrm{~L}_{8}(1200 \mathrm{mg} / \mathrm{kg}$ vitamin $\mathrm{C}$ and $8 \%$ lipid); $\mathrm{C}_{300} \mathrm{~L}_{12}(300 \mathrm{mg} / \mathrm{kg}$ vitamin $\mathrm{C}$ and $12 \%$ lipid $) ; \mathrm{C}_{600} \mathrm{~L}_{12}(600 \mathrm{mg} / \mathrm{kg}$ vitamin $\mathrm{C}$ and $12 \%$ lipid $) ;$ and $\mathrm{C}_{1200} \mathrm{~L}_{12}(1200 \mathrm{mg} / \mathrm{kg}$ vitamin $\mathrm{C}$ and $12 \%$ lipid).

b Vitamin and mineral mix (Socil Guyomarc' $\mathrm{H}^{\circledR}$ ): vitamin A, 1,200,000 UI; vitamin $\mathrm{D}_{3}, 200,000$ UI; vitamin E, $1200 \mathrm{mg}$; vitamin $\mathrm{K}_{3}, 2400 \mathrm{mg}$; vitamin $\mathrm{B}_{1}, 4800 \mathrm{mg}$; vitamin $\mathrm{B}_{2}, 4800 \mathrm{mg}$; vitamin $\mathrm{B}_{12}, 4800 \mathrm{mcg}$; vitamin $\mathrm{B}_{6}$, $4800 \mathrm{mg}$; vitamin C, 0; calcium D-pantothenate, $12,000 \mathrm{mg}$; niacin, 24,000 mg; folic acid, $1200 \mathrm{mg}$; biotin, $48 \mathrm{mg}$; choline chlorate, $108 \mathrm{~g}$; cobalt, $10 \mathrm{mg}$; cooper, $3000 \mathrm{mg}$; iron sulfate heptahydrate, 50,000 mg; iodine, $100 \mathrm{mg}$; manganese, 20,000 mg; selenium, $100 \mathrm{mg}$; zinc sulfate, 30,000 mg; carrier q.s.p., $1000 \mathrm{~g}$.

c Antioxidant.

d L-ascorbyl-2-polyphosphate, $35.0 \%$ vitamin C.

e Determined value.

f Calculated value.

\section{Phase I - Hematological Assay}

Blood samples were obtained after $112 \mathrm{~d}$. Six fish per treatment were randomly chosen and anesthetized with benzocaine at $1.0 \mathrm{~g} / 15 \mathrm{~L}$. Blood samples were collected from the caudal vein using tuberculin syringes rinsed with ethylenediaminetetraacetic acid (dipotassium salt PA 2-hydrate $3.0 \%$ ) to determine red blood cell (RBC) count, hematocrit (Htc), and hemoglobin $(\mathrm{Hb})$.

RBC counts were obtained in a hemocytometer with diluted whole blood. Htc was determined by the microhematocrit method and $\mathrm{Hb}$ by the cianometahemoglobin method using a standard kit (Labtest Diagnostica S.A.mega, Brazil). Mean corpuscular volume (MCV) and mean corpuscular $\mathrm{Hb}$ concentration (MCHC) were calculated. Blood analyses methodology was as per Jain (1986).

The same fish were bled again for glucose and cortisol analyses using heparin as anticoagulant. Plasma samples were collected following centrifugation of whole blood from six fish at $2000 \mathrm{~g}$. Plasma from two fish from the same treatment were pooled to obtain one composite sample. Glucose was determined using King and Garner's (1947) methodology and cortisol by DPC radioimmunoassay kit - Diagnostic Products Corporation (coat-a-count, solid-phase DPC, Brazil).

\section{Phase I - Measurement of Liver Content of Vitamin $C$}

Liver samples were obtained after $112 \mathrm{~d}$. Six fish per treatment were randomly chosen and 
sacrificed with high-dose anesthesia (benzocaine USP Synth). Livers were collected and immediately immersed in liquid nitrogen to avoid vitamin $\mathrm{C}$ oxidation and then stored frozen at $-80 \mathrm{C}$ for subsequent vitamin $\mathrm{C}$ concentration determination by HPLC - reverse phase as per Wang et al. (1988). Vitamin C concentration in the liver was determined in an HPLC equipped with diode array detector (SPDM10AVP; Shimadzu Labtec, Brazil), through a Supelcosil LC- $\mathrm{NH}_{2}$ column with $1.5 \mathrm{~mL} / \mathrm{min}$ constant flux and using a mobile phase $10 \mathrm{mM}$ acetonitril/sodium phosphate buffer, $\mathrm{pH} 2.6$ $(75 / 25)$ at $40 \mathrm{C}$.

\section{Phase II - Procedure}

After the above analyses, 48 fish were redistributed, in the same experimental structure, into three aquariums per treatment at a density of two fish per aquarium, six fish per treatment, with average weight of $105.13 \pm 19.71 \mathrm{~g}$. The water temperature was gradually dropped from 26.0 to $18.0 \mathrm{C}$ over $7 \mathrm{~d}$.

After this, fish were transferred to another experimental structure and randomly stocked into twenty-four $40 \mathrm{~L}$ aquariums at two fish per aquarium. Each aquarium was supplied with individual aeration and biofiltration. Room temperature was controlled at $18.0 \mathrm{C}$. The experimental design was the same as Phase I.

Fish were kept at this temperature for $25 \mathrm{~d}$ and then at $11.0 \mathrm{C}$ for another $11 \mathrm{~d}$. After this 36-d low-temperature stress period, the same blood parameters and vitamin $\mathrm{C}$ liver content as at the end of Phase I were evaluated.

\section{Statistical Analysis}

The statistical analysis used a two-way ANOVA technique for a $2 \times 3$ factorial experiment in a completely randomized design, with two additional treatments. The ANOVA was complemented with both the Scheffé multiple contrasts and the Tukey test for comparisons within the factorial structure (Zar 1999). Differences between means were reported as significant if $P<0.05$. Variables in both phases were studied by repeated measurement to compare the mean profile of each treatment, with the construction of simultaneous confidence intervals (Johnson and Wichern 1992).

\section{Results}

Fish fed basal diet $\left(\mathrm{C}_{0} \mathrm{~L}_{0}\right)$ had significantly $(P<0.05)$ lower erythrocytes and Htc values than the average Phase I factorial treatments. $\mathrm{Hb}$ was lower, although not significantly different from factorial averages. After temperature stress, $\mathrm{C}_{0} \mathrm{~L}_{0}$ fish had lower blood values than at Phase I (Table 2). Fish fed vitamin C-supplemented diets showed a significant effect of this vitamin on erythrocyte number and Htc with a slight increase in $\mathrm{Hb}$. There was a linear increase for vitamin $\mathrm{C}$ supplementation, although 600 and $1200 \mathrm{mg} / \mathrm{kg}$ diet vitamin C was not significantly different.

There was a significant effect after lowtemperature stress for mean values of $\mathrm{MCV}$ and MCHC between blood from fish fed basal diet and those from the factorial groups. This was not seen between fish fed supplemented diet (factorial) and $\mathrm{C}_{125} \mathrm{~L}_{6}$. Vitamin $\mathrm{C}$ and lipid supplementation did not affect any of these hematimetric values, irrespective of supplementation level.

Table 3 shows the mean vitamin $\mathrm{C}$ liver concentration, plasmatic cortisol, and glucose levels in fish fed diets with different levels of vitamin C supplement and lipids submitted to low-temperature stress. Levels were significantly higher $(P<0.05)$ in fish fed vitamin $\mathrm{C}$ supplemented diets than in fish fed basal diet $\left(\mathrm{C}_{0} \mathrm{~L}_{0}\right)$ before stress. The $125 \mathrm{mg} / \mathrm{kg}$ vitamin C-supplemented diet also had a lower liver vitamin $\mathrm{C}$ concentration than other supplemented groups. Higher supplementation levels produced an average increase of $35.8 \%$ in liver concentration. Similar to blood values, increased liver vitamin $\mathrm{C}$ was related to diet vitamin $\mathrm{C}$, although there was no significant difference between 600 and $1200 \mathrm{mg} / \mathrm{kg}$ diets. Comparing the before and after stress values, there was a similar tendency in fish on no vitamin $\mathrm{C}$ supplementation and fish on 125-mg vitamin $\mathrm{C}$ supplementation.

There was a significant poststress effect on plasmatic cortisol on fish fed basal diet compared with factorial supplemented fed fish; no supplementation produced a similar plasmatic cortisol concentration before and after. However, 
TABLE 2. Mean values of erythrocytes (Erit), hematocrit (Htc), and hemoglobin (Hb) for Nile tilapia juveniles fed diets supplemented with different levels of vitamin $C$ and lipids and submitted to temperature stress. ${ }^{1}$

\begin{tabular}{|c|c|c|c|c|c|c|}
\hline Diets & $\begin{array}{c}\text { Erit Phase I } \\
(106 / \mu \mathrm{L})\end{array}$ & $\begin{array}{l}\text { Erit Phase II } \\
(106 / \mu \mathrm{L})\end{array}$ & $\begin{array}{c}\text { Htc Phase I } \\
(\%)\end{array}$ & $\begin{array}{c}\text { Htc Phase II } \\
(\%)\end{array}$ & $\begin{array}{l}\text { Hb Phase I } \\
\text { (g/dL) }\end{array}$ & $\begin{array}{l}\text { Hb Phase II } \\
(\mathrm{g} / \mathrm{dL})\end{array}$ \\
\hline $\mathrm{C}_{0} \mathrm{~L}_{0}$ & $1.67( \pm 0.17)$ & $1.52( \pm 0.04)$ & $27.10( \pm 1.20)$ & $26.50( \pm 1.45)$ & $7.75( \pm 0.75)$ & $7.01( \pm 1.35)$ \\
\hline $\mathrm{C}_{125} \mathrm{~L}_{6}$ & $1.92 \alpha( \pm 0.11)$ & $1.72 \beta( \pm 0.11)$ & $29.40 \alpha( \pm 0.92)$ & $26.20 \beta( \pm 1.21)$ & $8.33( \pm 0.58)$ & $8.54( \pm 1.16)$ \\
\hline $\mathrm{C}_{300} \mathrm{~L}_{8}$ & $1.94( \pm 0.14)$ & $1.80 \mathrm{~b}( \pm 0.05)$ & $28.26 \alpha( \pm 0.17)$ & $25.10 \mathrm{~b} \beta( \pm 1.02)$ & $8.17( \pm 0.56)$ & $7.50( \pm 1.02)$ \\
\hline $\mathrm{C}_{600} \mathrm{~L}_{8}$ & $2.09( \pm 0.20)$ & $1.95 \mathrm{a}( \pm 0.07)$ & $29.20 \alpha( \pm 0.29)$ & $27.60 \mathrm{a} \beta( \pm 0.80)$ & $7.65( \pm 0.33)$ & $7.70( \pm 1.49)$ \\
\hline $\mathrm{C}_{1200} \mathrm{~L}_{8}$ & $1.99( \pm 0.10)$ & $1.95 \mathrm{a}( \pm 0.06)$ & $30.27 \alpha( \pm 2.01)$ & $27.75 \mathrm{a} \beta( \pm 0.67)$ & $7.96( \pm 0.34)$ & $8.39( \pm 0.40)$ \\
\hline $\mathrm{C}_{300} \mathrm{~L}_{12}$ & $1.98( \pm 0.19)$ & $1.79 \mathrm{~b}( \pm 0.09)$ & $29.50 \alpha( \pm 0.82)$ & $25.00 \mathrm{~b} \beta( \pm 1.45)$ & $8.22( \pm 0.80)$ & $7.68( \pm 0.91)$ \\
\hline $\mathrm{C}_{600} \mathrm{~L}_{12}$ & $1.98( \pm 0.17)$ & $1.80 \mathrm{ab}( \pm 0.08)$ & $29.00 \alpha( \pm 0.77)$ & $27.08 \mathrm{a} \beta( \pm 0.88)$ & $8.00( \pm 0.49)$ & $8.02( \pm 2.37)$ \\
\hline $\mathrm{C}_{1200} \mathrm{~L}_{12}$ & $1.97( \pm 0.17)$ & $1.94 \mathrm{a}( \pm 0.05)$ & $29.17 \alpha( \pm 0.83)$ & $27.00 \mathrm{a} \beta( \pm 0.57)$ & $8.01( \pm 0.41)$ & $8.32( \pm 1.38)$ \\
\hline Cont. $\mathrm{I} \times$ fat. $^{2}$ & 0.01 & 0.05 & 0.05 & ns & ns & ns \\
\hline Cont. II $\times$ fat. $^{3}$ & $\mathrm{~ns}$ & $\mathrm{~ns}$ & $\mathrm{~ns}$ & ns & ns & ns \\
\hline \multicolumn{7}{|l|}{ Vitamin C effect } \\
\hline 300 & 1.96 & 1.80 & 28.88 & 25.05 & 8.20 & 7.59 \\
\hline 600 & 2.04 & 1.88 & 29.10 & 27.34 & 7.83 & 7.86 \\
\hline 1200 & 1.98 & 1.95 & 29.72 & 27.38 & 7.99 & 8.36 \\
\hline \multicolumn{7}{|l|}{ Lipid effect } \\
\hline \multirow[t]{3}{*}{$(P$ level $)$} & ns & ns & ns & ns & ns & ns \\
\hline & 2.01 & 1.90 & 29.24 & 26.82 & 7.92 & 7.86 \\
\hline & 1.98 & 1.84 & 29.22 & 26.36 & 8.08 & 8.01 \\
\hline $\begin{array}{l}\text { Vitamin } \mathrm{C} \times \text { lipid } \\
\quad(P \text { level })\end{array}$ & $\mathrm{ns}$ & $\mathrm{ns}$ & $\mathrm{ns}$ & ns & ns & ns \\
\hline
\end{tabular}

$\mathrm{ns}=$ not significant.

${ }^{1}$ Different lowercase letters indicate differences between vitamin C levels, different uppercase letters indicate differences between lipid levels, and different Greek letters indicate differences between Phase I and Phase II.

2 Control I $\left(\mathrm{C}_{0} \mathrm{~L}_{0}\right) \times$ mean of factorial treatments.

${ }^{3}$ Control II $\left(\mathrm{C}_{125} \mathrm{~L}_{6}\right) \times$ mean of factorial treatments.

${ }^{4} P$ level $=$ statistic significance.

the other treatments showed a $65.0 \%$ increase in plasmatic cortisol after stress. The 600 and $1200 \mathrm{mg}$ vitamin C supplemented diets showed a significant reduction in plasmatic cortisol. Vitamin C supplementation had no effect on plasmatic glucose concentration.

\section{Discussion}

Nile tilapia fed vitamin C- and lipid-deficient diet had lower blood values as previously reported by Feldman et al. (2000). This author stated that erythrocyte synthesis requires adequate concentrations of many nutrients including lipids, proteins, carbohydrates, minerals, and vitamins. Abnormal erythrocyte synthesis can be caused by deficiencies in these nutrients that are critical for RBC production. They also emphasized that, at the beginning, pituitary, adrenal and thyroid regulate erythrocyte synthesis by $(\mathrm{ACTH})$, growth hormone $(\mathrm{GH})$, epineph- rine, and norepinephrine which lead increased erythropoietin concentration stimulating RBCs production.

Decreased Htc in both nutrient absence and deficiency reported in channel catfish by Lim and Lovell (1978); rainbow trout, Oncorhynchus mykiss, Albrektsen et al. (1988) and hybrid tilapia, Oreochromis niloticus $\times$ Oreochromis aureus; Shiau and Jan (1992), was also seen in this study. Lim et al. (2000), however, reported that Htc was not affected by vitamin $\mathrm{C}$ levels.

Either vitamin $\mathrm{C}$ absence or deficiency can impair iron metabolism, affecting its distribution in the organism, which may lead to decreased RBC production (Albrektsen et al. 1988). According to Devlin (1997) vitamin C also facilitates the iron absorption by the intestine and its redistribution to different tissues, including $\mathrm{Hb}$ synthesis. 
TABLE 3. Mean vitamin C liver concentration, plasmatic cortisol, and glucose concentrations for Nile tilapia juveniles fed diets supplemented with different levels of vitamin $C$ and lipids and submitted to temperature stress. ${ }^{1}$

\begin{tabular}{|c|c|c|c|c|c|c|}
\hline Diets & $\begin{array}{c}\text { Vitamin C } \\
\text { Phase I } \\
(\mu \mathrm{g} / \mathrm{g})\end{array}$ & $\begin{array}{c}\text { Vitamin C } \\
\text { Phase II } \\
(\mu \mathrm{g} / \mathrm{g})\end{array}$ & $\begin{array}{l}\text { Cortisol } \\
\text { Phase I } \\
(\mathrm{mg} / \mathrm{dL})\end{array}$ & $\begin{array}{l}\text { Cortisol } \\
\text { Phase II } \\
(\mathrm{mg} / \mathrm{dL})\end{array}$ & $\begin{array}{l}\text { Glucose } \\
\text { Phase I } \\
(\mathrm{mg} / \mathrm{dL})\end{array}$ & $\begin{array}{l}\text { Glucose } \\
\text { Phase II } \\
(\mathrm{mg} / \mathrm{dL})\end{array}$ \\
\hline $\mathrm{C}_{0} \mathrm{~L}_{0}$ & $120.85 \alpha( \pm 13.60)$ & $18.90 \beta( \pm 1.63)$ & $30.65( \pm 3.19)$ & $29.07( \pm 7.94)$ & $33.19( \pm 4.27)$ & $32.46( \pm 3.68)$ \\
\hline $\mathrm{C}_{125} \mathrm{~L}_{6}$ & $313.18 \alpha( \pm 19.60)$ & $20.93 \beta( \pm 4.49)$ & $15.91( \pm 7.80)$ & $10.27( \pm 6.75)$ & $32.48( \pm 7.28)$ & $33.41( \pm 4.37)$ \\
\hline $\mathrm{C}_{300} \mathrm{~L}_{8}$ & $280.19 \mathrm{~b} \alpha( \pm 24.30)$ & $27.77 \mathrm{~b} \beta( \pm 1.18)$ & $15.97( \pm 17.79)$ & $11.56( \pm 1.99)$ & $40.70( \pm 5.82)$ & $69.59( \pm 38.15)$ \\
\hline $\mathrm{C}_{600} \mathrm{~L}_{8}$ & $480.39 \mathrm{a} \alpha( \pm 46.90)$ & $33.65 \mathrm{a} \beta( \pm 4.87)$ & $22.43 \alpha( \pm 5.86)$ & $9.62 \beta( \pm 3.26)$ & $34.09( \pm 4.31)$ & $65.51( \pm 37.20)$ \\
\hline $\mathrm{C}_{1200} \mathrm{~L}_{8}$ & $510.00 \mathrm{a} \alpha( \pm 32.90)$ & $30.19 \mathrm{a} \beta( \pm 1.41)$ & $34.20 \alpha( \pm 8.83)$ & $8.49 \beta( \pm 3.94)$ & $35.94( \pm 8.47)$ & $32.57( \pm 2.67)$ \\
\hline $\mathrm{C}_{300} \mathrm{~L}_{12}$ & $340.43 \mathrm{~b} \alpha( \pm 63.70)$ & $27.05 \mathrm{~b} \beta( \pm 1.81)$ & $20.91( \pm 20.26)$ & $13.80( \pm 17.73)$ & $33.85( \pm 6.50)$ & $32.35( \pm 5.11)$ \\
\hline $\mathrm{C}_{600} \mathrm{~L}_{12}$ & $450.52 \mathrm{a} \alpha( \pm 70.18)$ & $32.80 \mathrm{a} \beta( \pm 2.52)$ & $30.06 \alpha( \pm 6.48)$ & $10.81 \beta( \pm 3.08)$ & $37.19( \pm 4.48)$ & $33.03( \pm 4.76)$ \\
\hline $\mathrm{C}_{1200} \mathrm{~L}_{12}$ & $490.47 \mathrm{a} \alpha( \pm 60.18)$ & $34.55 \mathrm{a} \beta( \pm 4.53)$ & $21.63 \alpha( \pm 9.80)$ & $6.75 \beta( \pm 6.09)$ & $43.14( \pm 6.80)$ & $43.89( \pm 16.06)$ \\
\hline Cont. $I \times$ fat $^{2}$ & 0.05 & 0.05 & ns & 0.05 & ns & ns \\
\hline Cont. II $\times$ fat $^{3}$ & 0.05 & 0.05 & ns & ns & ns & ns \\
\hline \multicolumn{7}{|l|}{ Vitamin C effect } \\
\hline$(P$ level $)$ & 0.05 & 0.05 & $\mathrm{~ns}$ & ns & ns & ns \\
\hline 300 & 310.31 & 27.41 & 18.44 & 12.68 & 37.28 & 50.97 \\
\hline 600 & 465.46 & 33.23 & 26.25 & 10.12 & 35.64 & 49.27 \\
\hline 1200 & 500.24 & 32.37 & 27.92 & 7.62 & 39.54 & 38.23 \\
\hline \multicolumn{7}{|l|}{ Lipid effect } \\
\hline \multirow[t]{3}{*}{$(P$ level $)$} & $\mathrm{ns}$ & $\mathrm{ns}$ & $\mathrm{ns}$ & $\mathrm{ns}$ & $\mathrm{ns}$ & ns \\
\hline & 423.53 & 30.54 & 24.20 & 9.89 & 36.91 & 55.89 \\
\hline & 427.14 & 31.47 & 24.20 & 10.45 & 38.06 & 36.42 \\
\hline $\begin{array}{l}\text { Vitamin } \mathrm{C} \times \text { lipid } \\
\quad(P \text { level })\end{array}$ & $\mathrm{ns}$ & $\mathrm{ns}$ & ns & ns & $\mathrm{ns}$ & $\mathrm{ns}$ \\
\hline
\end{tabular}

ns $=$ not significant.

${ }^{1}$ Different lowercase letters indicate differences between vitamin $\mathrm{C}$ levels; different uppercase letters indicate differences between lipid levels; and different Greek letters indicate differences between Phase I and Phase II.

2 Control I $\left(\mathrm{C}_{0} \mathrm{~L}_{0}\right) \times$ mean of factorial treatments.

${ }^{3}$ Control II $\left(\mathrm{C}_{125} \mathrm{~L}_{6}\right) \times$ mean of factorial treatments.

${ }^{4} P$ level $=$ statistic significance.

Although $\mathrm{Hb}$ concentration was not affected by vitamin $\mathrm{C}$ supplementation before or after temperature stress, $\mathrm{Hb}$ levels were lower (Table 2) in fish fed basal diet than those supplemented showing that they could be depleting their organic reserves, which could lead to anemia. The lack of significance in initial $\mathrm{Hb}$ values could be because of an insufficient feeding period (Phase I) and also sufficient diet iron and hepatic vitamin $\mathrm{C}$ concentration (Table 3 ). Soliman et al. (1994) reported that the absence of vitamin $\mathrm{C}$ in diets for hybrid tilapia led to low Htc, low liver vitamin C concentration, decreased growth, poor feed conversion ratio, and low protein efficiency.

After low-temperature stress fish fed vitamin C-deficient diet had difficulty maintaining erythrocyte synthesis, as in Phase I, revealed by an $18.7 \%$ decrease in erythrocytes and 11.7\% decrease in Hb. Pickering (1981) and Wedemeyer et al. (1990) describe physiological changes during stress due to catecholamines and adrenocorticotropic hormones including energy reserve mobilization and an increase in blood pressure and oxygen concentration. Based on results in Table 2, it can be seen that vitamin C supplementation facilitates iron absorption, the most important $\mathrm{Hb}$ component, mitigating the effect of stress, and keeping the cellular membrane intact because of its antioxidant action. This allowed vitamin E to prevent the oxygen reactive species action on unsaturated fatty acids, thus avoiding reduced erythrocyte membrane resistance with possible hemolysis. Also, Waagbo et al. (1993) observed a significant increase in $\mathrm{Hb}$ concentration related to increased vitamin $\mathrm{C}$ supplement in Atlantic salmon, Salmo salar.

Analyzing blood parameters both before and after low-temperature stress (Table 2), vitamin C supplementation maintained them at appropriate levels after stress, although a significant decrease in Htc could have been caused by low $\mathrm{Hb}$ and erythrocytes. Hattingh and Van Pletzen 
(1974) also observed decreased Htc in Labeo umbratus after $3 \mathrm{~d}$ of capture stress.

This may demonstrate the body's attempt to maintain blood cell synthesis, releasing immature cells with higher corpuscular volume, and consequently lower $\mathrm{Hb}$ concentration. Barros et al. (2002) observed that high vitamin C supplementation in diet resulted in higher Htc. However, this increase was also a result of higher corpuscular volume. Hrubec et al. (2000) determined normal values (115.0 and 183.0 fL) of MCV for hybrid tilapia. Although our results seem to be appropriate, the poststress results in vitamin $\mathrm{C}$-deficient fish $(\mathrm{MCV}=174.34 \mathrm{fL})$ were close to the highest value determined by this author.

Although absence of vitamin C supplementation had determined a significantly lower vitamin $\mathrm{C}$ concentration in the liver (Table 3), it could still be considered high as none was added by diet. As fish liver results from the vitamin Cdeficient diet do not correspond to the diet analysis, it could not be explained. The importance of vitamin $\mathrm{C}$ supplement concentrations higher than necessary to avoid deficiency and to determine a good reserve was described by Durve and Lovell (1982). Similarity between the 600 and $1200 \mathrm{mg}$ vitamin C results could be explained by possible liver saturation, demonstrating that $600 \mathrm{mg} / \mathrm{kg}$ vitamin $\mathrm{C}$ in diet is the cost-effective level for maintaining organic reserves and that $125 \mathrm{mg} / \mathrm{kg}$ vitamin $\mathrm{C}$ in diet and below was not sufficient to maintain liver concentrations after low-temperature stress. The minimum liver concentrations of vitamin C for rainbow trout (Hilton et al. 1978) and channel catfish (Lim and Lovell 1978) were 20.0 and $30.0 \mu \mathrm{g} / \mathrm{g}$, respectively. Although there was no fish mortality after low-temperature stress, in intensive culture systems where homeostasis is continually threatened and vitamin $\mathrm{C}$ reserves were redistributed, fish may not resist cumulative stress as a result of low vitamin $\mathrm{C}$ reserve, thus leading to mortality. The possible decrease in tissue vitamin $\mathrm{C}$ concentration after long periods of stress and the possible impairment in immune responses were observed by $\mathrm{Li}$ et al. (1994, 1998).

Poststress decreased cortisol concentrations in fish fed vitamin C-supplemented diets
(Table 3) could be explained by Kitabchi (1967) who proposed that high levels of ascorbic acid have an inhibitory role in steroid synthesis, by preventing the conversion of unsaturated fatty acid into cholesterol esters, which are incorporated into steroids. Consequently, vitamin C supplementation could mitigate the effect of stress. Similar results were obtained by Ortuno et al. (2002) with Sparus aurata. Studies with common carp, Cyprinus carpio (Dabrowska et al. 1991), channel catfish (Li et al. 1998), and Atlantic salmon (Davies et al. 1998) failed to detect the positive effects of vitamin $\mathrm{C}$ supplementation in plasmatic cortisol levels after stress.

Although this study showed no significant effect from lipids on cortisol and glucose levels (Table 3) before or after stress, Barton (1997) observed that increased fatty acids gave fish better resistance to stress because of high energetic mobilization. Barton et al. (1988) showed that Oncorhynchus tshawytscha fed a diet supplemented with high levels of energy had the highest glucose response to handling, suggesting better resistance. From this we can infer that in our study fish fed of diets supplemented with high lipid levels could be more prepared to cope with low-temperature stress because of their organic reserves. There was a similarity between glucose values before and after temperature stress. Schreck (2000) emphasized that fish were able to compensate and acclimatize to stress conditions as a result of behavioral and physiological changes. However, there is a metabolic cost linked to this compensation that can impair growth, reproduction, and resistance.

The physiological responses can not be considered as only resulting from low-temperature stress. There was additional adaptation as fish were transferred from one experimental facility to another. Although the situation describes a cumulative stress, the extended period could facilitate homeostasis with different stress hormone concentrations. Certainly, in these challenging situations, glycogen reserves may not be sufficient to maintain energy demand, which could require the use of organic reserves mainly because of cortisol action. Survival results suggest good physiological conditions attained by 
supplemented vitamin $\mathrm{C}$ and lipid diet. The physiological results from fish fed a vitamin C- and lipid-deficient diet indicated that an extended period of stress would certainly result in mortality.

\section{Conclusion}

Results from this study show that the absence of vitamin C supplementation impairs RBC formation and does not enable fish to cope with stress, vitamin $\mathrm{C}$ supplement above species requirement is an efficient way to mitigate stress and $600 \mathrm{mg}$ of vitamin $\mathrm{C}$ supplement is an economic and physiologically sufficient way to prepare fish to cope with low-temperature stress, and lipid supplementation does not impact stress biochemical parameters.

\section{Acknowledgments}

We are grateful to the Fundação de Amparo à Pesquisa do Estado de São Paulo - FAPESP, Brazil, and to SOCIL GUYOMARC'H INDÚSTRIA E COMÉRCIO Ltda for supporting this research.

\section{Literature Cited}

Albrektsen, S., O. Lie, and K. Sanders. 1988. Ascorbyl palmitate as a dietary vitamin $\mathrm{C}$ source for rainbow trout (Salmo gairdneri). Aquaculture 71:359-365.

AOAC (Association of Official Analytical Chemists). 2000. Official methods of analysis of the Association of Official Analytical Chemists, 17th edition. AOAC, Gaithersburg, Maryland, USA.

Barros, M. M., L. E. Pezzato, G. K. Kleemann, H. Hisano, and G. J. M. Rosa. 2002. Níveis de vitamina $\mathrm{C}$ e ferro para tilápia do Nilo (Oreochromis niloticus). Revista Brasileira de Zootecnia 31:2149-2156.

Barton, B. A. 1997. Stress in finfish: past, present and future - a historical perspective. Pages 1-33 in G. K. Iwana, A. D. Pickering, J. P. Sumpter, and C. B. Schreck, editors. Fish stress and health in aquaculture. University Press, Cambridge.

Barton, B. A., C. B. Schreck, and L. G. Fowler. 1988. Fasting and diet content affect stress-induced changes in plasma glucose and cortisol in juvenile chinook salmon. Progressive Fish-Culturist 50:16-22.

Dabrowska, H., K. Dabrowski, K. Meyer-Burgdorff, W. Hanke, and K. D. Gunther. 1991. The effect of large doses of vitamin $\mathrm{C}$ and magnesium on stress responses in common carp (Cyprinus carpio). Comparative Biochemistry and Physiology. 89b:539-545.
Davies, K. B., B. A. Simco, M. Li, and E. Robinson. 1998. Effect of reduction of supplemental dietary vitamins on the stress response of channel catfish (Ictalurus punctatus). Journal of the World Aquaculture Society 29:319-324.

Devlin, T. M. 1997. Manual de Bioquímica com Correlações Químicas. Edgard Blucher, São Paulo, Brazil.

Durve, V. S. and R. T. Lovell. 1982. Vitamin C and disease resistance in channel catfish (Ictalurus punctatus). Canadian Journal of Fisheries and Aquatic Sciences. 39:948-951.

Feldman, B. F., J. G. Zinkl, and N. C. Jain. 2000. Schalm's veterinaty hematology, 5th edition. Lippincott Williams \& Wilkins, Philadelphia, Pennsylvania, USA.

Gamperl, A. K., M. Wilkinson, and R. G. Boutilier. 1994. $\beta$-Adrenoreceptors in the trout (Oncorhynchus mykiss) heart: characterization, quantification \& effects of repeated catecholamine exposure. General Comparative Endocrinology. 95:259-272.

Hattingh, J. and A. J. J. Van Pletzen. 1974. The influence of capture and transportation on some blood parameters of freshwater fish. Comparative Biochemistry and Physiology 49:607-609.

Hilton, J. W., C. Y. Cho, and S. J. Slinger. 1978. Effect of graded levels of supplemental ascorbic acid in practical diets of rainbow trout. Journal of the Fisheries Research Board of Canada 35:431-436.

Hrubec, T. C., J. L. Cardinale, and S. A. Smith. 2000. Hematology and plasma chemistry reference intervals for cultured Tilapia (Oreochromis hybrid). Veterinary Clinical Pathology 29:618-623.

Jaffa, M. 1989. Vitamin C can curb those stress associated losses. Fish Farming International 12:18-19.

Jain, N. C. 1986. Schalm's veterinary hematology, 4th edition. Lea \& Febiger, Philadelphia, Pennsylvania, USA.

Johnson, R. A. and D. W. Wichern. 1992. Applied multivariate statistical methods, 3rd edition. PrenticeHall, Englewood Cliffs, New Jersey, USA.

King, E. J. and R. J. Garner. 1947. Colorimetric determination of glicose. Journal of Clinical Pathology. 1:1-30.

Kitabchi, A. E. 1967. Ascorbic acid in steroidogenesis. Nature 215:1385-1386.

Lemly, A. D. 1996. Wastewater discharges may be most hazardous to fish during winter. Environmental Pollution 32:169-174.

Li, M. H. and R. T. Lovell. 1985. Elevated levels of dietary ascorbic acid increase immune responses in channel catfish. Journal of Nutrition. 115:123-31.

Li, M. H. and E. H. Robinson. 1994. Effect of dietary vitamin $\mathrm{C}$ on tissue vitamin $\mathrm{C}$ concentration in channel catfish, Ictalurus punctatus, and clearance rate at two temperatures - a preliminary investigation. Journal Applied Aquaculture. 4:59-71.

Li, M. H., D. J. Wise, and E. H. Robinson. 1998. Effect of dietary vitamin $\mathrm{C}$ on weight gain, tissue ascorbate 
concentration, stress response, and disease resistance of channel catfish Ictalurus punctatus. Journal of the World Aquaculture Society. 29:1-8.

Lim, C. and R. T. Lovell. 1978. Pathology of vitamin C deficiency syndrome in channel catfish (Ictalurus punctatus). Journal of Nutrition 108:1137-1146.

Lim, C., P. H. Klesius, M. H. Li, and E. H Robinson. 2000. Interaction between dietary levels of iron and vitamin $\mathrm{C}$ on growth, hematology, immune response and resistance of channel catfish (Ictalurus punctatus) to Edwardsiella ictaluri challenge. Aquaculture 185:313-327.

Lovell, R. T. and B. Sirikul. 1974. Winter feeding of channel catfish. 28:208-216.

Ortuno, J., M. A. Esteban, and J. Meseguer. 2002. The effect of dietary intake of vitamins $\mathrm{C}$ and $\mathrm{E}$ on the stress response of gilthead seabream (Sparus aurata L.). Fish Shellfish Immunology 12:1-12.

Pickering, A. D. 1981. Introduction: the concept of biological stress. Pages 1-9 in A.D. Pickering, editor. Stress and fish. Academic Press, London, UK.

Schreck, C. B. 2000. Accumulation and long term effects of stress in fish. Pages 147-158 in G. P. Moberg and J. A. Mench, editors. The biology of animal stress. CAB International, Wallingford, UK.

Sealey, W. M., C. Lim, and P. H. Klesius. 1997. Influence of the dietary level of iron from iron methionine and iron sulfate on immune response and resistance of channel catfish to Edwardsiella ictaluri. Journal of the World Aquaculture Society 28:142-149.

Shiau, S. Y. and F. L Jan. 1992. Dietary ascorbic acid requirement of juvenile tilapia Oreochromis niloticus $x$ O. aureus. Fisheries Science. 58:671-675.

Soliman, A. K., K. Jauncey, and R. J. Roberts. 1994. Water-soluble vitamin requirements of tilapia: ascorbic acid (vitamin C) requirement of Nile tilapia, Oreochromis niloticus (L.). Aquaculture and Fisheries Management 25:269-278.

Waagbo, R., J. Glette, E. Raa-Nilsen, and K. Sandnes. 1993. Dietary vitamin C, immunity and disease resistance in Atlantic salmon (Salmo salar). Fish Physiology and Biochemistry 12:61-73.

Wang, X. Y., L. Ming-Long, T. H. Hung, and P. A. Seib. 1988. Liquid chromatographic determination of L-ascorbate 2-polyphosphate in fish feeds by enzymatic release of L-ascorbate. Journal - Association of Official Analytical Chemists 71:1158-1161.

Wedemeyer, G. 1969. Stress induced ascorbic acid depletion and cortisol production in two salmonid fish. Comparative Biochemistry and Physiology 29:1247-1251.

Wedemeyer, G. A., B. A. Barton, and D. J. Mcleay. 1990. Stress and acclimation. Pages 451-489 in C. B. Schreck and P. B. Moyle, editors. Methods for fish biology. American Fisheries Society, Bethesda, Maryland, USA.

Zar, J. H. 1999. Biostatistical analysis, 4th edition. Prentice-Hall, Englewood Cliffs, New Jersey, USA. 